\title{
The Effect of Diabetes Mellitus on the Ventricular Epicardial Activation and Repolarization in Mice
}

\author{
M. A. VAYKSHNORAYTE ${ }^{1}$, A. O. OVECHKIN ${ }^{1,2}$, J. E. AZAROV ${ }^{1,3}$ \\ ${ }^{1}$ Laboratory of Cardiac Physiology, Institute of Physiology, Komi Science Center, Ural Branch, \\ Russian Academy of Sciences, Syktyvkar, Russia, ${ }^{2}$ Department of Internal Diseases, Komi Branch \\ of Kirov State Medical Academy, Syktyvkar, Russia, ${ }^{3}$ Department of Physiology, Komi Branch of \\ Kirov State Medical Academy, Syktyvkar, Russia
}

Received July 22, 2011

Accepted April 10, 2012

On-line June 6, 2012

\section{Summary}

Cardiac repolarization is prolonged in diabetes mellitus (DM), however the distribution of repolarization durations in diabetic hearts is unknown. We estimated the ventricular repolarization pattern and its relation to the ECG phenomena in diabetic mice. Potential mapping was performed on the anterior ventricular surface in healthy $(n=18)$ and alloxan-induced diabetic $(n=12)$ mice with the 64-electrode array. Activation times, end of repolarization times, and activation-recovery intervals (ARIs) were recorded along with limb lead ECGs. ARIs were shorter in the left as compared to right ventricular leads $(P<0.05)$. The global dispersion of repolarization, interventricular and apicobasal repolarization gradients were greater in DM than in healthy animals $(P<0.03)$. The increased dispersion of repolarization and apicobasal repolarization gradient in DM correlated with the prolonged QTC and Tpeak-Tend intervals, respectively. The increased ventricular repolarization heterogeneity corresponded to the electrocardiographic markers was demonstrated in DM.

\section{Key words}

Activation-recovery interval - Diabetes mellitus - Dispersion of repolarization • Mouse heart $\bullet$ Tpeak-Tend

\section{Corresponding author}

M. A. Vaykshnorayte, Laboratory of Cardiac Physiology, Institute of Physiology, Komi Science Center, Ural Division, Russian Academy of Sciences, Pervomayskaya st., 50, Syktyvkar, Russia, 167982. Tel/fax: +78212240085. E-mail: m.vaykshnorayte@mail.ru

\section{Introduction}

Diabetes mellitus (DM) causes a number of cardiovascular effects such as the activation of oxidative processes, necrosis and apoptosis of cardiomyocytes, collagen synthesis, endothelial dysfunction, enhanced atherogenesis, and vascular remodeling (Fang et al. 2004). Obesity, dyslipidemia, arterial hypertension, and coronary heart disease associated with DM affect the cardiovascular system as well. Diabetic cardiomyopathy defined as the lesion of the heart independent of the ischemic and hypertensive agents (Rubler et al. 2007) largely caused by the metabolic and/or autonomic alterations is responsible for the higher prevalence of ventricular arrhythmias in DM patients independently of the presence of arterial hypertension, coronary atherosclerosis, and heart failure (Movahed et al. 2007). On the other hand, a paradoxically increased resistance to the ischemic and reperfusion lesions has been demonstrated in the early stages of DM (Ravingerová et al. 2000).

Cardiac electrophysiological consequences of DM have been described. The diabetic hearts demonstrate prolonged action potential durations (Magyar et al. 1992, Shimoni et al. 1994, Lengyel et al. 2007, Howarth et al. 2007, 2009) resulted from the downregulation or remodeling of the repolarizing ionic currents: $\mathrm{I}_{\mathrm{to}}$ (Magyar et al. 1992, Lengyel et al. 2007, Howarth et al. 2009), $\mathrm{I}_{\mathrm{Kr}}$ (Howarth et al. 2009), or $\mathrm{I}_{\mathrm{Ks}}$ (Lengyel et al. 2007). However, little is known about how this prolongation of repolarization is distributed throughout the myocardium, 
i.e. whether the repolarization gradients are altered in DM. Furthermore, the above mentioned myocardial electrophysiological effects of DM have been largely observed in isolated tissues, whereas these effects could be modulated by the altered autonomic tone, hemodynamic load etc in vivo.

The body surface potential distribution during ventricular repolarization is also affected in DM with potential maximum and minimum being lower in diabetic patients (Zdárská et al. 2007). Among other changes, the increase in the QT duration and dispersion associated with the alteration of repolarization durations have been demonstrated to be the major electrocardiographic predictors of proarrhythmic myocardial changes in DM (Rutter et al. 2002). However, the prognostic potential of the QT dispersion measurements has been subjected to criticism on the basis of the prospective clinical trials (Zabel et al. 1998, Brendorp et al. 2001) and from the theoretical viewpoint (Malik and Batchvarov 2000). Instead, the Tpeak-Tend interval (Tp-e) as a novel repolarization dispersion index was proposed (Yan and Antzelevitch 1998). Although the data on the electrophysiological nature of Tp-e remain contradictory (e.g., it is unclear which myocardial characteristic underlies the Tp-e: either the transmural (Yan and Antzelevitch 1998) or the global (Opthof et al. 2007) dispersion of repolarization), this electrocardiographic measure appears promising as a predictor of ventricular arrhythmias and/or mortality in different conditions (Lellouche et al. 2007, Haarmark et al. 2009). However, in the DM setting, Tp-e has not been evaluated so far.

The objective of the present study was to estimate the repolarization pattern on the anterior ventricular surface and its relation to the body surface electrocardiographic indices of ventricular repolarization, such as QT and Tp-e, in diabetic mice.

\section{Methods}

The investigation conforms with the Guide for the Care and Use of Laboratory Animals published by the US National Institutes of Health (NIH Publication No. 85-23, revised 1996). Experiments were done on a total of 47 mice of either sex aged 5 months. 29 animals were given a single subcutaneous injection of alloxan (40 mg/100 g body weight) in order to induce type $1 \mathrm{DM}$. 13 mice died of the DM complications within two weeks after the alloxan injection and DM did not developed in 4 animals. As a result, the DM group consisted of
12 mice ( 8 males and 4 females) at the follow-up of 4-5 weeks and 18 animals serve as controls. The plasma glucose concentration was measured in the blood withdrawn from the caudal vena with the One Touch glucometer (LifeScan, Inc., USA). DM was ascertained with the fasting plasma glucose concentration more than $7 \mathrm{mmol} / \mathrm{l}$. The characteristics of the normal and DM animals are given in Table 1.

Table 1. Characteristics of the animals of the control and DM groups (Me; $25 \% ; 75 \%$ ).

\begin{tabular}{cccc}
\hline & $\begin{array}{c}\text { FPG, } \\
\mathbf{m m o l} / \mathbf{l}\end{array}$ & $\begin{array}{c}\text { Body } \\
\text { weight, } \\
\mathbf{g}\end{array}$ & $\begin{array}{c}\text { Heart } \\
\text { weight, } \\
\mathbf{g}\end{array}$ \\
\hline $\begin{array}{c}\text { Control } \\
\left(\begin{array}{l}n=18) \\
D M\end{array}\right.\end{array}$ & $6.6(5.8 ; 6.7)$ & $28(26 ; 32)$ & $0.16(0.15 ; 0.18)$ \\
$(n=12)$ & $8.4(7.7 ; 11.5)^{*}$ & $30(28 ; 32)$ & $0.15(0.14 ; 0.16)$ \\
\hline
\end{tabular}

* $\mathrm{p}<0.05$ vs controls. DM, diabetes mellitus; FPG, fasting plasma glucose concentration.

The mice were anesthetized with zoletil (10 mg/kg body weight, i.m.), intubated and ventilated artificially. The conventional ECG was recorded in limb leads. After the ECG recording, the heart was exposed via midsternal incision. The temperature of $37.5-38.0{ }^{\circ} \mathrm{C}$ in the mediastinum was maintained in the course of the experiment by the heating of the room air and the irrigation with warm saline. The potential mapping was performed with the electrode array being a rectangular 64-electrode matrix $5 \times 5 \mathrm{~mm}$. Unipolar electrograms were recorded in reference to Wilson's central terminal. Data acquisition was done by means of a custom-designed mapping system (16 bits; bandwidth 0.05 to $1000 \mathrm{~Hz}$; sampling rate $4000 \mathrm{~Hz}$ ).

The QRS, QT, Tp-e, and T wave durations were measured in the II limb lead at display screen sweep of 100 to $1300 \mathrm{~mm} / \mathrm{s}$ and a voltage gain of $0.02 \mathrm{mV} / \mathrm{mm}$. As QT measurements are often accompanied by errors associated with the determination of the end of the $\mathrm{T}$ wave two blinded observers have made repeated measurements of QT and Tp-e intervals. To assess the intra- and interobserver variabilities we used Wilcoxon test and Bland-Altman plot to evaluate agreement between two measurements. The measurements done by the same observer on different days demonstrated no 
significant differences in QT interval (the values are given for the combined group of healthy and diabetic animals): 49.5 (41.8; 62.0) vs. 51.5 (45.3; 54.5) ms, $\mathrm{p}=0.62$ and S.D. of the difference was $6.8 \mathrm{~ms}$ or $13.1 \%$ of the Mean. The difference between the data of the first and the second observers was also insignificant: 49.5 (44.9; 56.8) vs. $51.5(43.8 ; 60.5) \mathrm{ms}, \mathrm{p}=0.49$ and S.D. of the difference $7.3 \mathrm{~ms}$ or $13.5 \%$ of the Mean. The measurements of the Tp-e interval have shown the similar rate of intra- and interobserver reproducibility, because the variabilities of the Tp-e as well as QT values were predominantly associated with the determination of the Tend point. The corrected QT (QTc) was calculated as $\mathrm{QTc}=\mathrm{QT}+0.384(1000-\mathrm{RR})$ (Karjalainen et al. 1994).

In each epicardial lead, the activation time (AT) and the end of repolarization time (RT) were determined as $\mathrm{dV} / \mathrm{dt}$ min during $\mathrm{QRS}$ and $\mathrm{dV} / \mathrm{dt} \max$ during ST-T, respectively. The determinations were done automatically, inspected by the blinded observer and corrected if necessary. The activation-recovery interval (ARI) was measured as the difference between RT and AT (Millar et al. 1985) and served as an index of local repolarization duration. The dispersions of ATs, ARIs, and RTs were determined as the differences between the maximal and minimal values of each variable. The interventricular and apicobasal gradients of RTs were taken as the differences between the average RTs obtained from the appropriate leads of the epicardial electrode array: Apicobasal RT gradient $=$ average $\mathrm{RT}$ (basal leads) - average RT (apical leads); Interventricular RT gradient $=$ average RT (right ventricular leads) average RT (left ventricular leads). Similarly, interventricular and apicobasal gradients of ARIs and ATs were calculated as well.

Statistical examination was done with SPSS 11.5 software (SPSS, USA). The data are given as median and interquartile interval [Me $(25 \% ; 75 \%)]$. The Wilcoxon test for paired comparisons and Mann-Whitney test for the comparisons between the diabetic and control groups were used. The differences were considered significant at $\mathrm{P}<0.05$. The assessment of epicardial measures as the independent predictors of QTc and Tp-e duration was done by a multiple linear regression (enter method).

\section{Results}

Diabetic and control mice did not differ in heart rate and QRS durations. On the other hand, Tp-e, QT and QTc durations were longer in the animals with DM (Table 2).

The activation sequence of the studied epicardial patch on the anterior ventricular surface was from the right ventricle to the left ventricle both in control and diabetic mice. However, the duration of the activation (activation dispersion) of this area was longer in DM group (Table 3). Similarly, the ATs measured in respect to the QRS onset were longer in diabetic animals. The average RTs as well as average ARIs did not distinguish between the healthy and diabetic animals. However, when the right and left ventricular leads were considered separately, a significant RT prolongation was seen on the right ventricle in DM group as compared to controls. The ARI duration was inversely related to the ATs $(r=-0.66$ and $\mathrm{r}=-0.54$ in control and DM group, respectively) indicating that the earlier activated areas tended to show prolonged repolarization and vice versa. As a result, the end of repolarization sequence was opposite to the activation sequence and directed from the left ventricle to the right ventricle in both groups (Fig. 1).

Table 2. Electrocardiographic variables in the control and DM groups (Me; $25 \% ; 75 \%$ ).

\begin{tabular}{llll}
\hline & $\begin{array}{l}\text { Control } \\
(\mathbf{n = 1 8})\end{array}$ & $\begin{array}{l}\text { DM } \\
(\mathbf{n = 1 2})\end{array}$ & $\boldsymbol{P}$ \\
\hline$Q R S, m s$ & $9(8 ; 10)$ & $10(9 ; 10)$ & 0.199 \\
$Q T, m s$ & $46.5(41.5 ; 50.0)$ & $57.5(52.8 ; 69.8)$ & 0.002 \\
QTc, $m s$ & $364(359.8 ; 364.8)$ & $392.1(387.6 ; 397.5)$ & 0.005 \\
Tpeak-Tend, $\mathrm{ms}_{\text {Heart rate, } \mathrm{min}^{-1}}$ & $32.5(29.3 ; 37.8)$ & $42.5(39.5 ; 51.8)$ & 0.001 \\
\hline
\end{tabular}

DM, diabetes mellitus. 
Table 3. Ventricular epicardial activation and repolarization measures (Me; $25 \% ; 75 \%$ ).

\begin{tabular}{|c|c|c|c|}
\hline & $\begin{array}{l}\text { Control } \\
(n=18)\end{array}$ & $\begin{array}{l}\text { DM } \\
(n=12)\end{array}$ & $P$ \\
\hline \multicolumn{4}{|l|}{ Average } \\
\hline$A T, m s$ & $6(6 ; 7)$ & $9(7 ; 9)$ & 0.012 \\
\hline$A R I, m s$ & $40(37 ; 49)$ & $44(38 ; 47)$ & 0.567 \\
\hline$R T, m s$ & $47(44 ; 56)$ & $53(48 ; 55)$ & 0.678 \\
\hline \multicolumn{4}{|c|}{ Right ventricle } \\
\hline$A T, m s$ & $6(5 ; 7)$ & $8(7 ; 9)$ & 0.01 \\
\hline$A R I, m s$ & $43(40 ; 51)$ & $49(47 ; 56)$ & 0.11 \\
\hline$R T, m s$ & $49(46 ; 57)$ & $57(55 ; 63)$ & 0.03 \\
\hline \multicolumn{4}{|c|}{ Left ventricle } \\
\hline$A T, m s$ & $7(6 ; 9) *$ & $9(8 ; 10) *$ & 0.008 \\
\hline$A R I, m s$ & $39(34 ; 47) *$ & $40(35 ; 44) *$ & 0.88 \\
\hline$R T, m s$ & $45(42 ; 55) *$ & $49(45 ; 53) *$ & 0.56 \\
\hline \multicolumn{4}{|c|}{ Interventricular gradient } \\
\hline$A T, m s$ & $1(0.6 ; 1)$ & $1(0.7 ; 2)$ & 0.65 \\
\hline$A R I, m s$ & $5(4 ; 6)$ & $10(8 ; 16)$ & $<0.001$ \\
\hline$R T, m s$ & $4(2 ; 5)$ & $8(7 ; 13)$ & $<0.001$ \\
\hline \multicolumn{4}{|l|}{ Apex } \\
\hline$A T, m s$ & $6.6(5.6 ; 7.6)$ & $8.5(7.5 ; 9.2)$ & 0.036 \\
\hline$A R I, m s$ & $42.1(37.0 ; 49.5)$ & $43.3(39.1 ; 47.7)$ & 0.751 \\
\hline$R T, m s$ & $48.0(45.3 ; 56.3)$ & $51.5(47.9 ; 56.8)$ & 0.434 \\
\hline \multicolumn{4}{|l|}{ Base } \\
\hline$A T, m s$ & $6.7(6.0 ; 8.3)$ & $8.9(8.2 ; 9.5)$ & 0.008 \\
\hline$A R I, m s$ & $40.1(37.2 ; 51.9)$ & $45.3(42.7 ; 55.8)$ & 0.197 \\
\hline$R T, m s$ & $47.6(44.5 ; 59.3)$ & $55.2(51.3 ; 63.2)$ & 0.117 \\
\hline \multicolumn{4}{|c|}{ Apicobasal gradient } \\
\hline$A T, m s$ & $0.7(-0.4 ; 1.5)$ & $0.7(0.1 ; 1.3)$ & 0.789 \\
\hline$A R I, m s$ & $-0.8(-2.9 ; 1.0)$ & $0.6(-0.4 ; 2.9)$ & 0.112 \\
\hline$R T, m s$ & $-0.3(-1.1 ; 0.2)$ & $0.9(-0.1 ; 2.5)$ & 0.026 \\
\hline \multicolumn{4}{|c|}{ Dispersions } \\
\hline$A T, m s$ & $4(3 ; 5)$ & $4(3 ; 6)$ & 0.180 \\
\hline$A R I, m s$ & $13(11 ; 16)$ & $26(13 ; 30)$ & 0.002 \\
\hline$R T, m s$ & $11(8 ; 14)$ & $23(14 ; 27)$ & 0.008 \\
\hline
\end{tabular}

* $\mathrm{p}<0.05$ vs right ventricle; ARI, activation-recovery interval; AT, activation time; DM, diabetes mellitus; RT, end of repolarization time.

A significant interventricular gradient in ATs, ARIs, and RTs was found both in control and DM groups (Table 3) with the ATs being longer in the left ventricular leads, and ARIs and RTs being longer in the right ventricular leads. The interventricular differences in ATs were similar in both groups, whereas the repolarization gradients both of ARIs and RTs were greater in diabetic mice as compared to controls, which resulted from the increased ARIs and RTs on the right ventricular surface. Apicobasal differences in activation and repolarization measures (Table 3) were not apparent in the healthy animals, whereas a slight but significant RT difference was observed in the DM group. Consequently, the calculated apicobasal gradient of RTs was significantly greater in the diabetic mice.

The contribution of the epicardial repolarization measures to the ECG repolarization measures was estimated in the combined (control and DM) group with the multiple linear regression model. The global RT dispersion was demonstrated to be a significant 


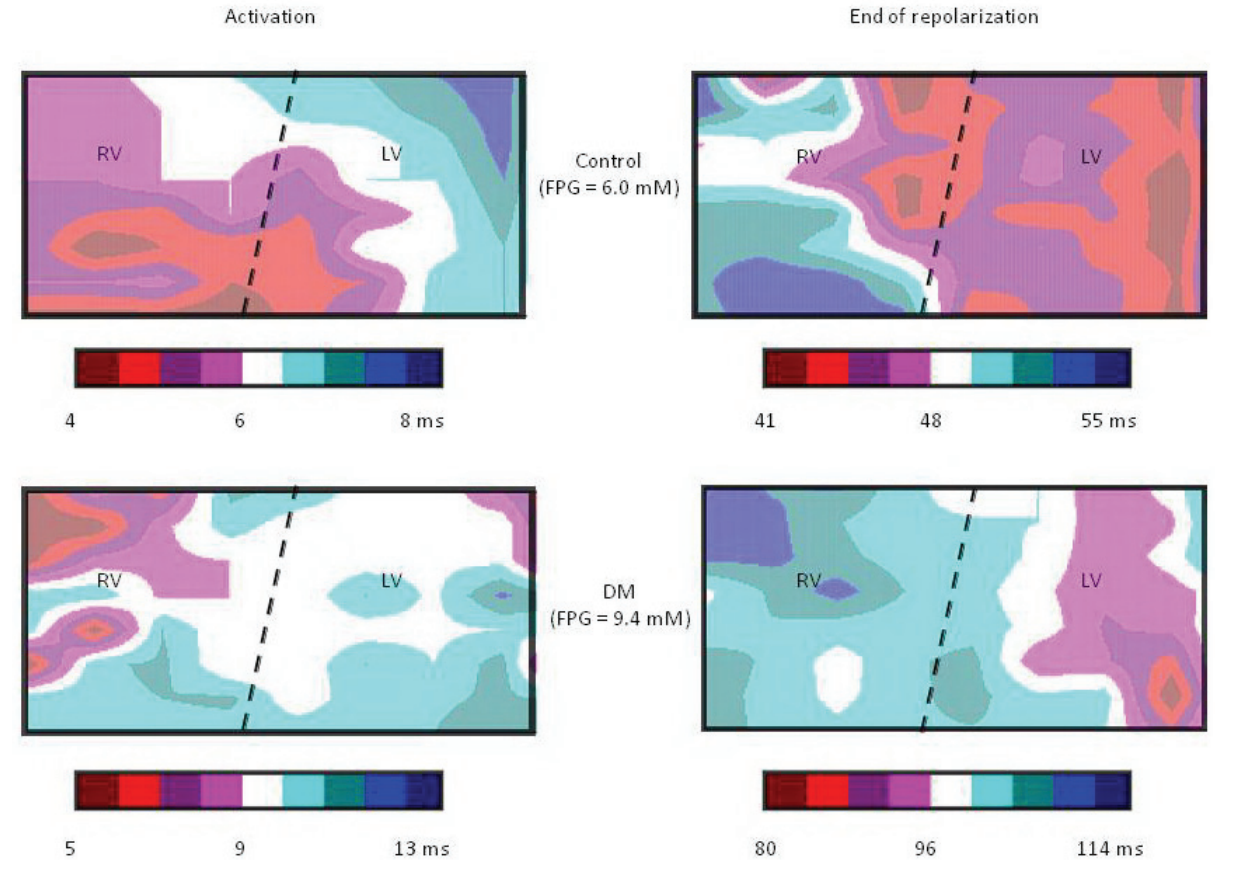

Fig. 1. The representative isochronal maps of activation (left column) and end of repolarization (right column) sequences of the anterior ventricular surface of the healthy (upper row) and diabetic (lower row) animals. See opposite activation and end of repolarization sequences both in control and DM maps. The RTs were generally prolonged in DM, though the statistical significance was shown only for the RV leads. The scales indicate the ATs and RTs with respect to the QRS onset. The dashed lines identify for the interventricular grooves. AT, activation time; DM, diabetes mellitus; FPG, fasting plasma glucose concentration; LV, left ventricle; RT, end of repolarization time; RV, right ventricle.
Table 4. Beta coefficients for the independent variables entered in the multiple regression models of the QTC and Tp-e duration in the body surface ECG.

\begin{tabular}{lcc}
\hline Variables & $\begin{array}{c}\mathbf{Q T c}(\mathbf{n}=\mathbf{3 0}), \\
\mathbf{R}=\mathbf{0 . 5 3} ; \\
\mathbf{R}^{\mathbf{2}=\mathbf{0 . 2 8}}\end{array}$ & $\begin{array}{c}\mathbf{T p}-\mathbf{e}(\mathbf{n}=\mathbf{3 0}), \\
\mathbf{R}=\mathbf{0 . 5 8}, \\
\mathbf{R}^{\mathbf{2}}=\mathbf{0 . 3 3}\end{array}$ \\
\hline $\begin{array}{l}\text { Apicobasal RT } \\
\text { gradient } \\
\text { Interventricular } R T\end{array}$ & -0.11 & $0.54^{*}$ \\
$\begin{array}{l}\text { gradient } \\
\text { Global } R T \\
\text { dispersion }\end{array}$ & -0.05 & 0.11 \\
\hline
\end{tabular}

${ }^{*} \mathrm{p}<0.05$. R, multiple correlation coefficient and $\mathrm{R}^{2}$, determination coefficient for all independent variables.

independent predictor of the QTc duration and the apicobasal RT gradient was shown to be a significant independent predictor of the Tp-e duration (Table 4).

\section{Discussion}

The major findings of the present study were as follows: i) on the anterior ventricular surface in mice, repolarization was shorter in the left ventricular leads as compared to the right ventricular leads; ii) the interventricular and apicobasal gradients of repolarization timing were greater in the DM group than in healthy animals; similarly, the dispersions of repolarization
(ARIs and RTs) were increased in DM; iii) the epicardial indices of the larger extent of repolarization heterogeneity in diabetic mice were manifested in the increased body surface ECG intervals such as QTc and Tp-e durations.

One of the most consistent findings concerning the repolarization process in the cardiomyocytes from the diabetic heart is reported to be the prolongation of action potential duration (Magyar et al. 1992, Shimoni et al. 1994, Lengyel et al. 2007, Howarth et al. 2007, 2009). In the present study of the hearts in situ, we observed no significant prolongation of average ARI values within the mapped area on the anterior ventricular surface in DM mice which could be due to the relatively fast sinus rhythm, and relatively short follow-up period of DM. According to the published data (Nygren et al. 2007), the alteration of the activation process of the diabetic heart ventricles is more subtle. However, in the present study, we observed the indications of the ventricular activation slowing in mice with DM, specifically, the prolongation of ATs measured in respect to the QRS onset implying the slow impulse propagation from the conduction system to the mapped area.

In spite of the absence of the significant alterations of average repolarization durations in DM group, the distribution of repolarization durations and endings in the DM animals differed from that in the controls. The RTs on the right ventricular surface were longer in diabetic animals than in controls. This resulted from the significant increase in AT combined with the slight ARI prolongation of borderline significance in the 
right ventricular leads. Furthermore, the interventricular gradients in ARIs and RTs were greater in the DM group reflecting a nonuniform changes in the repolarization properties of the left and right ventricles in DM. Mice represent a specific animal model as the major repolarizing current is $I_{t o, f}$ which is predominantly expressed in the right ventricle (Brunet et al. 2004) where the prolongation of action potential duration was found in mice with DM (Aomine and Yamato 2000, Szebeni et al. 2009) implying the role of $I_{t o, f}$ in diabetic electrophysiological remodeling. This point has been further supported by the fact that the action potential duration in diabetic mice lost 4-aminopyridine sensitivity (Aomine and Yamato 2000). In the streptozotocin rat model of DM, Howarth et al. (2007) found the prolongation of action potential duration in the right ventricle but not in the left ventricle. On the other hand, the changes in the repolarization process of the left ventricular myocardium was observed in other studies (Magyar et al. 1992, Lengyel et al. 2007, Howarth et al. 2009). The apicobasal gradient of RTs was also greater in the diabetic hearts suggesting the nonuniform alteration of the repolarization process across the ventricles. The relatively slight difference in repolarization timing between the apex and base was presumably due to the fact that the utmost apical and basal areas were not covered by the electrode array.

Our findings suggest that the electrocardiographic expression of the observed DM effects on ventricular repolarization was the prolongation of the QT, QTc and Tp-e. There is a large body of controversy concerning the meaning of the repolarization ECG phenomena. Specifically, the duration of the Tp-e have been ascribed to the magnitude of either transmural (Yan and Antzelevitch 1998) or apicobasal (Xia et al. 2005) gradients in repolarization timing. The data of the present study demonstrated that the increase of Tp-e in the body surface ECG in DM were associated with the increase of the interventricular and apicobasal repolarization gradient. The multiple regression analysis suggested that in spite of the relatively small magnitude, it was the apicobasal difference in the end of repolarization times that contribute significantly to the Tp-e. It should be taken into account, however, that the transmural repolarization gradient was not studied in the present investigation and therefore, its contribution to the ECG phenomena should not be excluded. However, with the aid of the simultaneous measurements of body surface potential distributions, transmural and epicardial repolarization patterns, our recent study (Vaykshnorayte et al. 2011) demonstrated that the transmural repolarization profiles contribute little if any to the body surface $\mathrm{T}$ wave potentials. Thus, we could suggest that the increase of the Tp-e interval in the setting of DM was caused by the increase of the apicobasal repolarization gradient.

In our experimental model, we found the evidence for the development of the proarrhythmic substrate in the hearts of diabetic animals. Within the mapped area, the ATs as well as the dispersions of both ARIs and RTs were higher in the DM group. The electrocardiographic expression of the increased dispersion of repolarization was demonstrated with multiple regression model to be QTc duration. The prolongation of ATs measured in respect to the QRS onset indirectly indicated the activation slowing. The increased dispersion of repolarization predisposing to the unidirectional conduction block has long been recognized as a proarrhythmic condition (Han and Moe 1964, Burton and Cobbe 2001). Both findings suggested the higher susceptibility of the diabetic heart to the reentrant arrhythmia. However, no spontaneous ventricular arrhythmic events were seen in the animals during the mapping procedure. Moreover, the published data on the arrhythmic susceptibility in DM are rather equivocal as DM was reported to be associated on one hand with the high prevalence of ventricular fibrillation independent of coronary artery disease or congestive heart failure (Movahed et al. 2007) and on the other hand with the decreased risk of ventricular fibrillation related to ischemia-reperfusion (Ravingerová et al. 2000). Coronel et al. (2006) underscored the fact that the mere increase in the dispersion of repolarization did not necessarily lead to reentry. Our findings indicated that the ventricular myocardium of the diabetic heart was predisposed to the reentrant arrhythmia by the activation slowing and the repolarization dispersion increase while a necessary precipitating event was lacking in our specific experimental setting. The association of the increased repolarization dispersion, apicobasal and interventricular repolarization gradients, Tp-e QT, and QTc intervals could provide an electrocardiographic estimate of the arrhythmogenic myocardial substrate in the DM setting.

\section{Limitations of the study}

In the present study, the durations of repolarization were measured from the extracellular electrograms. This method has been verified recently (Coronel et al. 2006), however, the obtained data should be taken cautiously due to the indirect nature of the 
measurements. Only the anterior ventricular surface was mapped, therefore, the results might have been modified, had the entire myocardium been studied. On the other hand, the same electrode array was utilized throughout the study and hence, the mutual electrode positions and interelectrode spacings were identical in the both groups that favored the comparisons between healthy and diabetic animals. The follow-up period in the present study was relatively short and the advanced effects of DM were not studied.

\section{Conclusion}

Thus, the interventricular and apicobasal repolarization gradients as well as repolarization dispersion within the anterior ventricular surface were greater in the diabetic mice as compared with the normal animals. The differences in the ventricular repolarization pattern were expressed in the increased Tp-e and QTc intervals in the body surface electrocardiogram in mice with DM.

\section{Conflict of Interest}

There is no conflict of interest.

\section{Acknowledgements}

The study was supported by the Ural Branch of the Russian Academy of Sciences (Project No 12-И-4-2059).

\section{References}

AOMINE M, YAMATO T: Electrophysiological properties of ventricular muscle obtained from spontaneously diabetic mice. Exp Anim 49: 23-33, 2000.

BRENDORP B, ELMING H, JUN L, KOBER L, MALIK M, JENSEN GB, TORP-PEDERSEN C: QT dispersion has no prognostic information for patients with advanced congestive heart failure and reduced left ventricular systolic function. Circulation 103: 831-842, 2001.

BRUNET S, AIMOND F, LI H, GUO W, ELDSTROM J, FEDIDA D, YAMADA KA, NERBONNE JM: Heterogeneous expression of repolarizing, voltage-gated $\mathrm{K}^{+}$currents in adult mouse ventricles. $J$ Physiol 559: 103-120, 2004.

BURTON FL, COBBE SM: Dispersion of ventricular repolarization and refractory period. Cardiovasc Res 50: 10-23, 2001.

CORONEL R, DE BAKKER JM, WILMS-SCHOPMAN FJ, OPTHOF T, LINNENBANK AC, BELTERMAN CN, JANSE MJ: Monophasic action potentials and activation recovery intervals as measures of ventricular action potential duration: experimental evidence to resolve some controversies. Heart Rhythm 3: 1043-1050, 2006.

CORONEL R, WILMS-SCHOPMAN FJ, OPTHOF T, JANSE MJ: Dispersion of repolarization and arrhythmogenesis. Heart Rhythm 6: 537-543, 2009.

FANG ZY, PRINS JB, MARWICK TH: Diabetic cardiomyopathy: evidence, mechanisms, and therapeutic implications. Endocr Rev 25: 543-567, 2004.

HAARMARK C, HANSEN PR, VEDEL-LARSEN E, PEDERSEN SH, GRAFF C, ANDERSEN MP, TOFT E, WANG F, STRUIJK JJ, KANTERS JK: The prognostic value of the Tpeak-Tend interval in patients undergoing primary percutaneous coronary intervention for ST-segment elevation myocardial infarction. J Electrocardiol 42: 555-560, 2009.

HAN J, MOE GK: Nonuniform recovery of excitability in ventricular muscle. Circ Res 14: 44-60, 1964.

HOWARTH FC, AL-SHARHAN R, AL-HAMMADI A, QURESHI MA: Effects of streptozotocin-induced diabetes on action potentials in the sinoatrial node compared with other regions of the rat heart. Mol Cell Biochem 300: 3946, 2007.

HOWARTH FC, JACOBSON M, QURESHI MA, SHAFIULLAH M, HAMEED RS, ZILAHI E, AL HAJ A, NOWOTNY N, ADEGHATE E: Altered gene expression may underlie prolonged duration of the QT interval and ventricular action potential in streptozotocin-induced diabetic rat heart. Mol Cell Biochem 328: 57-65, 2009.

KARJALAINEN J, VIITASALO M, MANTTARI M, MANNINEN V: Relation between QT intervals and heart rates from 40 to 120 beats/min in rest electrocardiograms of men and a simple method to adjust QT interval values. J Am Coll Cardiol 23: 1547-1553, 1994. 
LELlOUCHE N, De DIEGO C, AKOPYAN G, BOYLE NG, MAHAJAN A, CESARIO DA, WIENER I, SHIVKUMAR K: Changes and predictive value of dispersion of repolarization parameters for appropriate therapy in patients with biventricular implantable cardioverter-defibrillators. Heart Rhythm 4: 1274-1283, 2007.

LENGYEL C, VARRÓ A, TÁBORI K, PAPP JG, BACZKÓ I: Combined pharmacological block of I(Kr) and I(Ks) increases short-term QT interval variability and provokes torsades de pointes. Br J Pharmacol 151: 941-951, 2007.

MAGYAR J, RUSZNÁK Z, SZENTESI P, SZÛCS G, KOVÁCS L: Action potentials and potassium currents in rat ventricular muscle during experimental diabetes. J Mol Cell Cardiol 24: 841-853, 1992.

MALIK M, BATCHVAROV VN: Measurement, interpretation and clinical potential of QT dispersion. $J$ Am Coll Cardiol 36: 1749-1766, 2000.

MILLAR CK, KRALIOS FA, LUX RL: Correlation between refractory periods and activation-recovery intervals from electrograms: effects of rate and adrenergic interventions. Circulation 72: 1372-1379, 1985.

MOVAHED MR, HASHEMZADEH M, JAMAL M: Increased prevalence of ventricular fibrillation in patients with type 2 diabetes mellitus. Heart Vessels 22: 251-253, 2007.

NYGREN A, OLSON ML, CHEN KY, EMMETT T, KARGACIN G, SHIMONI Y: Propagation of the cardiac impulse in the diabetic rat heart: reduced conduction reserve. J Physiol 580: 543-560, 2007.

OPTHOF T, CORONEL R, WILMS-SCHOPMAN FJ, PLOTNIKOV AN, SHLAPAKOVA IN, DANILO P JR, ROSEN MR, JANSE MJ: Dispersion of repolarization in canine ventricle and the electrocardiographic $T$ wave: Tp-e interval does not reflect transmural dispersion. Heart Rhythm 4: 341-350, 2007.

RAVINGEROVÁ T, STETKA R, PANCZA D, ULICNÁ O, ZIEGELHÖFFER A, STYK J: Susceptibility to ischemiainduced arrhythmias and the effect of preconditioning in the diabetic rat heart. Physiol Res 49: 607-616, 2000.

RUBLER S, DLUGASH J, YUCEOGLU YZ, KUMRAL T, BRANWOOD AW, GRISHMAN A: New type of cardiomyopathy associated with diabetic glomerulosclerosis. Am J Cardiol 8: 595-602, 1972.

RUTTER MK, VISWANATH S, MCCOMB JM, KESTEVEN P, MARSHALL SM: QT prolongation in patients with Type 2 diabetes and microalbuminuria. Clin Auton Res 12: 366-372, 2002.

SHIMONI Y, FIREK L, SEVERSON D, GILES W: Short-term diabetes alters $\mathrm{K}^{+}$currents in rat ventricular myocytes. Circ Res 74: 620-628, 1994.

SZEBENI A, FALUS A, KECSKEMÉTI V: Electrophysiological characteristics of heart ventricular papillary muscles in diabetic histidine decarboxylase knockout and wild-type mice. J Interv Card Electrophysiol 26: 155-158, 2009.

VAYKSHNORAYTE MA, AZAROV JE, TSVETKOVA AS, VITYAZEV VA, OVECHKIN AO, SHMAKOV DN: The contribution of ventricular apicobasal and transmural repolarization patterns to the development of the T wave body surface potentials in frogs (Rana temporaria) and pike (Esox lucius). Comp Biochem Physiol A Mol Integr Physiol 159: 39-45, 2011.

XIA Y, LIANG Y, KONGSTAD O, LIAO Q, HOLM M, OLSSON B, YUAN S: In vivo validation of the coincidence of the peak and end of the $\mathrm{T}$ wave with full repolarization of the epicardium and endocardium in swine. Heart Rhythm 2: 162-169, 2005.

YAN GX, ANTZELEVITCH C: Cellular basis for the normal T wave and the electrocardiographic manifestations of the long-QT syndrome. Circulation 98: 1928-1932, 1998.

ZABEL M, KLINGENHEBEN T, FRANZ MR, HOHNLOSER SH: Assessment of QT dispersion for prediction of mortality or arrhythmic events after myocardial infarction: results of a prospective, long-term follow-up study. Circulation 97: 2543-2550, 1998.

ZDÁRSKÁ D, PELÍSKOVÁ P, CHARVÁT J, SLAVÍCEK J, MLCEK M, MEDOVÁ E, KITTNAR O: ECG body surface mapping (BSM) in type 1 diabetic patients. Physiol Res 56: 403-410, 2007. 